\title{
Crossroads
}

\author{
Crossroads | Walter Hill | 1986
}

Silvina Luzzi*

Universidad de Buenos Aires

Recibido: 10 de enero 2017; aceptado: 20 de febrero 2017

\begin{abstract}
Resumen
Un joven y virtuoso estudiante de guitarra clásica sueña en consagrarse como músico de blues. Para ello se propone encontrar una "melodía perdida” escrita por el mítico Robert Johnson. El film presenta una variante de la leyenda del pacto con el diablo y resulta útil para pensar la naturaleza del acto creador. Este artículo, basado en los aportes de Arnold Schoenberg, establece las diferencias entre un artesano y un artista, aportando una hipótesis sobre el acontecimiento musical.
\end{abstract}

Palabras clave: Schoenberg $\mid$ artista | acontecimiento | música

Crossroads

Abstract

A young person and virtuous southern guitar student dreams about becoming a blues musician. To achieve his goal, he defies himself to find a "lost melody" written by the mythical Robert Johnson.The film presents a variant of the legend of the agreement with the devil and it turns out useful to think the nature of the creating act. This article based on Arnold Schoenberg's contributions, establishes the differences between a craftsman and an artist, contributing a hypothesis on the musical event.

Keywords: Schoenberg | Artist | Event | music

El film Crossroads fue estrenado en nuestro país en 1986 bajo el título de "Encrucijada". Ralph Macchio encarna el personaje de Eugene Martone, un joven y virtuoso estudiante de guitarra clásica quien sueña en consagrarse como músico. Para esto posee un plan que lo llevará a la fama: encontrar una "melodía perdida" escrita nada menos que por un grande del blues: Robert Leroy Johnson.

Eugene se encuentra con Willie Brown, personaje encarnado por Joe Seneca, un viejo músico de blues quien reconoce rápidamente el talento y la ambición del joven. El viejo Willie se encuentra ya en el ocaso de su vida, una vida plena de anécdotas y aventuras con las que seduce a Eugene. Willie ha sabido sacarle el jugo a la vida, la ha vivido intensamente, pero en ese devenir contrajo una deuda que le quita el sueño y que debe saldar. Para esto debe volver "al origen”, en donde todo comenzó, al enigmático Mississippi.

Es así que en uno de sus encuentros el viejo Willie le confiesa a Eugene que él conoció a Robert Johnson, que compartieron escenario y que sabe cómo recuperar esa tan ansiada melodía perdida. Le promete revelarle el secreto a cambio de un favor: que lo lleve a Mississippi, lo que parece una modesta petición a cambio de la llave del éxito. Dice tener los medios para emprender el viaje pero que debido a su limitación física requiere de compañía. Eugene vacila pero finalmente acepta. Comienzan así a transitar una ruta llena de contratiempos pero nunca más “a tempo" para la vida del joven Eugene.

\section{El artesanado}

Un joven negro está parado en un cruce de caminos. En la escena siguiente se dirige a un departamento austero en el que un productor lo está esperando para grabar una canción. Comienza a cantar, acompañado por una guitarra un blues alusivo a un encuentro en "la encrucijada”. Este joven era Robert Leroy Johnson y esta escena corresponde a los inicios de su carrera.

*silvinaluzzi@yahoo.com.ar 


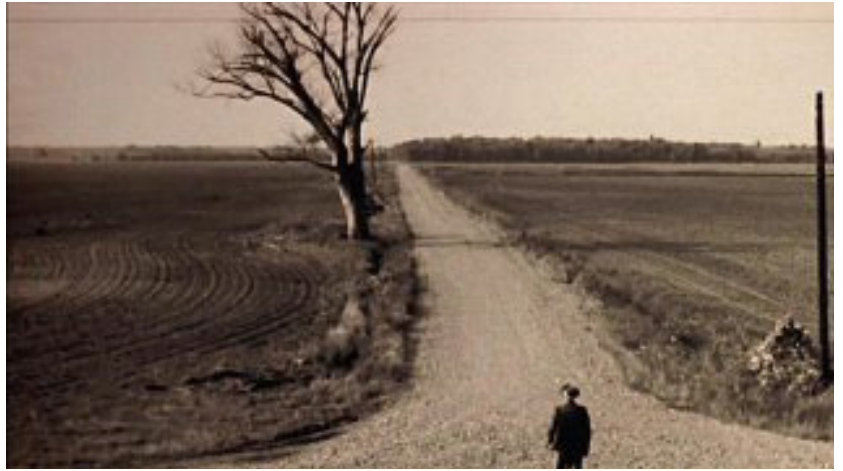

En la escena siguiente Eugene, escuchando este blues, queda cautivado por el llamado que Robert Johnson hace en la canción a un tal Willie Brown "ven a la encrucijada”. Indaga y advierte que Willie Brown está vivo y pasa sus días en un asilo. Raudamente se dirige hasta allí con la ilusión de que el viejo pueda darle los datos del Blues número 30 que lo llevará a la gloria pero, contra sus expectativas el viejo se rehúsa a atenderlo. Toma el puesto de personal de limpieza para poder acercarse a él y cuando finalmente consigue intercambiar escasas palabras en las que el supuesto Wiilie niega ser el personaje de la canción, ridiculizándolo.

En la escena siguiente Eugene se encuentra en un auditorio dando su clase de guitarra en la prestigiosa academia de música Julliart. Ejecuta de manera eximia una versión para guitarra de la Marcha Turca, de Mozart. Los estudiantes que están allí lo escuchan absortos hasta que Eugene termina su ejecución con una cadencia de blues. En una entrevista a solas con su maestro a causa de su irreverencia este le dice que es un estudiante prodigio pero que "no puede servir a dos amos", si persiste en otra práctica diferente a la clásica terminara perdiendo su talento.

\section{Servir a dos amos}

Eugene ama la música, ama tocar la guitarra y trabaja duro para ser un buen músico. Su alma vibra con blues, y alterna el estudio de los profesores tradicionales, con quienes él considera verdaderos maestros del blues, en especial Robert Johnson. En esta entrevista el profesor explicita cierta tensión de la que Eugene comienza a hacerse cargo.

¿Qué significa servir a dos amos? Para responder este primer interrogante que se abre a partir de la escena narrada y situar a nuestro personaje recurriremos a reflexiones que el músico, maestro y compositor Arnold
Schoenberg despliega en su tratado de armonía, publicado por primera vez en 1911. Dedicado al incomprendido Gustav Mahler, desarrolla de manera exhaustiva la armonía clásica.

Para Arnold Schoenberg la dicotomía que supuestamente enfrenta a la armonía clásica con otras posibles, no es tal. La armonía no existe como conocimiento intemporal, las estéticas no son más que gustos de época. Quienes promulgan a la armonía clásica como el paradigma de lo bello no hacen sino obstaculizar lo más valioso que guardan las obras de los grandes maestros: su impulso: lo pasado es materia de infinita plasticidad, aptas para recibir las más variadas formas (Schoenbreg,1979: xi). Al imperialismo de la estética contrapone la "búsqueda" ética.

De esta manera propone la emancipación de la disonancia. Los defensores de la armonía clásica, quienes la momifican y promulgan como ideal basan sus argumentos en la historia y en la naturaleza, ambas para Schoenberg base de sus propios argumentos a favor de la emancipación de las disonancias. La historia muestra cómo a una estética le ha sucedido otra, ¿por qué no pensar entonces que la armonía clásica tendrá también quien la suceda?

La naturaleza. En cuanto a la construcción del sonido contiene un gradiente de armónicos hasta ahora desechados por la armonía clásica dada su incapacidad para albergarlas. La diferencia entre consonancias y disonancias es, para este autor tan sólo una cuestión de grado.

"La materia de la música es el sonido. Deberá por tanto ser considerado en todas sus peculiaridades y efectos, capaz de engendrar arte, todas las sensaciones que provoca" (Schoenbreg, 1979: xv).

Partiendo desde estas tesis fuertes: el sonido debe ser considerado en todas sus formas y la estética como algo temporal. Lo pasado será entonces materia de infinita plasticidad, algo vivo, que guarda (mas allá de sus formas externas) en las obras de los grandes maestros lo que llama "impulso" -concepto asimilable al de "estilo", huella que el sujeto del acto plasma en la obra de arte (Ariel, 1979).

Retomando a nuestro personaje, Eugene, podríamos conjeturar que se encuentra desde los inicios del film en la primera encrucijada.

La sucesión de los hechos no ha sido casual. Luego de haber escuchado el llamado en aquel blues, busca a quien "ha devenido" maestro en absoluto desconocimiento de ambos. Ese final disruptivo del concierto de Mozart en 
Julliart otorga verdadero sentido a la ejecución y la meta de la obra de arte se revela estando en otro "lugarartesanado". Da comienzo así la verdadera "búsqueda”.

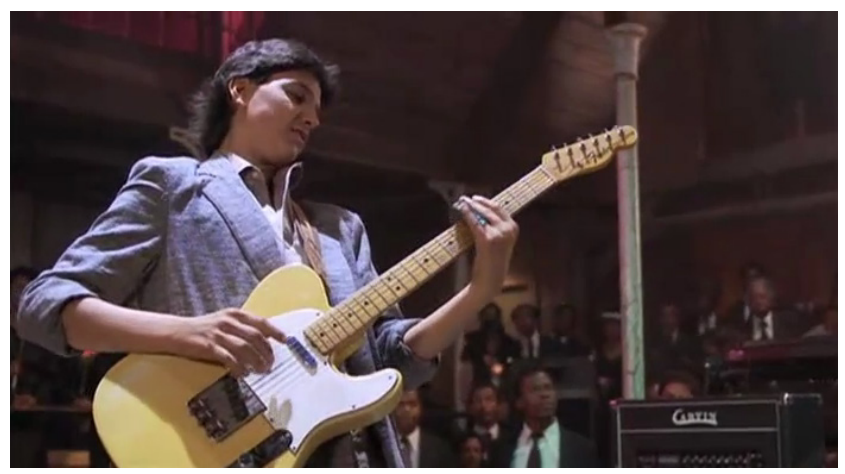

Necesitará para ello un verdadero maestro alguien capaz de guiarlo en este camino.

\section{¿Qué es un maestro?}

Comenzamos el apartado anterior haciendo referencia a la obra de Arnold Schoenberg "Tratado de Armonía” lo cual encierra una aparente contradicción. ¿Por qué quien ha proclamado la emancipación de la disonancia, el pasado como algo que ha de ser constantemente superado y el futuro imposible de ser enseñado, es el autor de uno de los libros de referencia en el mundo de la música sobre la armonía clásica?

Es que la enseñanza corresponde al artesanado:

El verdadero sentido y la meta de la obra de arte están en otro lugar más elevado y la enseñanza es impotente para alcanzar ese lugar. No por ello hay que proscribir la enseñanza musical, que puede ejercerse en dos estratos bien diferenciados. Primero: existe una suma de medios técnicos que hay que dominar, un repertorio de adecuaciones que hay que manejar con soltura (en caso de la armonía pueden sintetizarse en esto: obtener, disgregar, recobrar y suprimir la tonalidad con eficacia, teniendo siempre conciencia clara de los procedimientos empleados, de su valoración y de su grado de sencillez o complejidad), de manera que no se presenten obstáculos materiales. A esto llama Schöenberg "artesanado": es el "oficio", que consiste ante todo en acostumbrarse a conocer y a manipular el material de la música (el sonido). A este nivel la enseñanza puede ser eficaz si muestra al alumno un repertorio coherente de problemas y de posibles soluciones (Schoenberg, 1979: xv).

Coherente con esta visión de la pedagogía y la didáctica en la música considera que el maestro tendrá como objetivo propiciar el advenimiento de la agitación, motor de la vida del espíritu y de la creación artística. La relación entre el profesor y el alumno debe entenderse como una búsqueda conjunta en la que el profesor lo guiará hasta que éste, agitado espiritualmente, se separa de él para crear por sí mismo. El propio Schoenberg, frente a sus alumnos dice nunca haberse propuesto enseñar sólo lo que él sabía sino buscar lo que el alumno no sabía, obligándose a sí mismo a encontrar algo nuevo para cada uno de ellos.

Volviendo al film, hemos postulado la tesis de que Willie Brown se constituye como maestro de Eugene en absoluta ignorancia de ambos.

Luego de la entrevista en Julliart, Eugene vuelve al internado y logra finalmente la confirmación del músico, junto a la ratificación del desafío: si lo ayuda a fugarse y llegar hasta el mítico cruce de caminos, él le develará la canción por medio de la cual obtendrá la gloria en el mundo del blues. Eugene vacila pero finalmente accede. Esta decisión precipitada se explica por lo expuesto hasta el momento: el final del artesanado. Eugene sin saberlo, ha encontrado el maestro que lo guiará en la ruta de la "emancipación".

El viaje comienza con engaños y percances que sortear, pero ambos persisten. En el lugar y momento menos esperado comienza la primera lección. A lo lejos se escucha el sonido de un tren, su traqueteo, su bocina y Willie comienza a improvisar con su armónica Eugene lo escucha e intenta acoplarse. Es una melodía sencilla y él es un músico virtuoso pero su intento suena duro, disruptivo.

Siguen su ruta y Willie lo lleva hasta una casa de empeño con la premisa de que si quiere ser un bluesman necesita un instrumento acorde. Para pagar la compra debe dejar su antigua guitarra y el reloj que le ha regalado su madre en su cumpleaños. Esa guitarra que lo acompañó durante tantos años en Julliart es trocada por otra, que vibre en armonía al tránsito que hará a lo largo de este viaje.

Ocurren varias situaciones, entre ellas el encuentro amoroso de Eugene con una adolescente viajera quien se acopla al dúo, hasta que en una de sus andanzas y desconociendo las reglas del sur de los Estados Unidos, Eugene entra a un bar de negros. Esto es leído como una provocación de tal grado que Willie se ve obligado improvisar en el escenario invitándolo a Eugene a participar. A diferencia de la primera improvisación Eugene logra en este escenario hacer música con sus circunstanciales compañeros. 


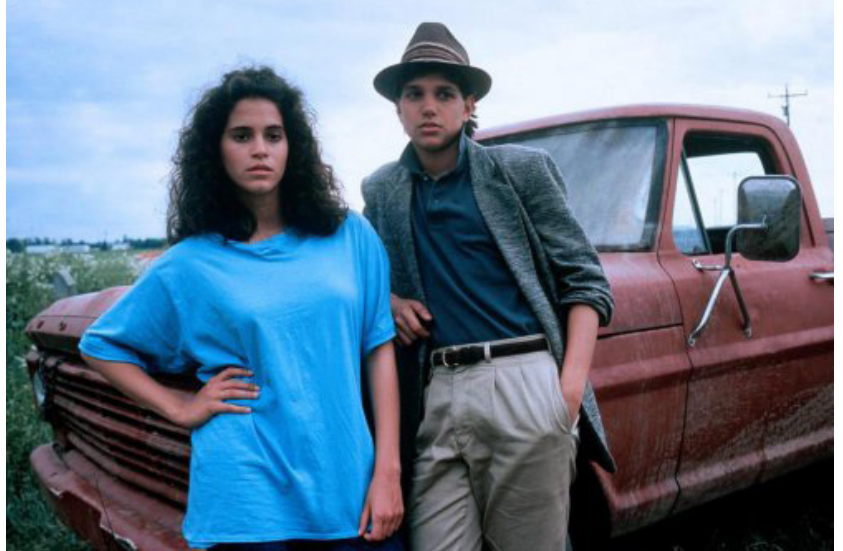

Así, poco a poco se van relativizado verdades a las que Eugene adscribía sin dudar. En cada nueva vivencia, se ha sorprendido de sí mismo y ha sido Willie, bajo la forma de viejo gruñón e irónico quien lo ha sostenido y acompañado. Este crecimiento interior se ve reflejado en su música. Tiene la técnica, el artesanado, y ahora también esa agitación producida por quien, como hemos postulado al comienzo, se ha constituido, sin saberlo como su maestro.

El recital es un éxito. Han recaudado dinero suficiente para dormir en un hotel. Esa noche ocurre el primer desengaño amoroso de nuestro personaje. Está triste y desconcertado, pero nuevamente surge allí la palabra de Willie que lo sostiene y le significa su dolor en relación al blues. Al mismo tiempo Willie atormentado por la proximidad de su encuentro con el pasado le confiesa que no existe la canción perdida. Johnson sólo escribió 29 blues, “deberás hacerla por ti mismo". Con esta afirmación da entrada a la incertidumbre del futuro de la que habla Arnold Schoenbreg, pieza clave para comprender el rol del maestro, impotente ante la empresa de la creación:

...la enseñanza no puede aportar medios ni procedimientos, técnicas ni estilos, porque lo que el alumno ha de hacer, precisamente, es inventar, es decir, ir contra mucho de lo que se le ha enseñado. ¿qué puede hacer aquí la pedagogía musical? Schöenberg contesta taxativamente: "la enseñanza que podría recibir un artista podría ser ante todo, ayudarle a escucharse a sí mismo" (Schoenberg, 1979: xv)

Han caído las garantías, la esperanza de la fórmula del éxito. Eugene, abatido pero íntegro, agitado entre la exaltación de aquel concierto y la pérdida de su primer amor, continúa al lado de Willie.

Todavía deben llegar a la encrucijada tal como lo pactaron al comienzo del viaje. Averiguaciones de por medio dan con el lugar. Ambos están parados en un cruce desolado. Ante la sorpresa de Eugene en medio de la nada aparece un auto conducido por un hombre negro que se detiene y saluda a Willie como si se hubieran conocido en el pasado. Ambos tienen una discusión en la que Willie le exige que se rompa el contrato. El hombre se ríe y se niega a hacerlo: su alma le pertenece y se volverán a ver nuevamente en el infierno a menos que encuentre un voluntario dispuesto a salvar su alma. Willie, desesperado intenta apartar a Eugene de la conversación pero el diablo no pierde la oportunidad de retarlo: harán un duelo de guitarras, si Eugene gana, el alma de Willie será liberada. Si pierde, se quedará con ambas.

Llegan al bar en el que se realizará el duelo. Eugene mira a su alrededor muerto de miedo. Willie, quien sabe de la seriedad de la situación y ve en los ojos el pánico de Eugene se acerca y le susurra "te doy toda la magia que poseo". Sube al escenario sin decir palabra alguna. Entre gritos y aplausos tocando la guitarra como amedrentándolo entra su contrincante -protagonizado por Steve Vay- Eugene se queda paralizado. Debe al menos igualar la actuación del candidato del diablo. Willie nuevamente acude en su auxilio, sube a escena y acompaña con la armónica para que Eugene logre dar rienda suelta a su talento. Habían recorrido un largo camino juntos en encuentros y desencuentros. Eugene ya no era el mismo que partió de Julliart. Curiosamente se encuentra batiéndose a duelo, ¿pero, qué es lo que duela en ese lance? Tal vez el final del camino de la niñez a la vida adulta, de la inmadurez a la madurez, de las certezas al abismo, a la incertidumbre del futuro, en síntesis, del artesanado al acto creador.

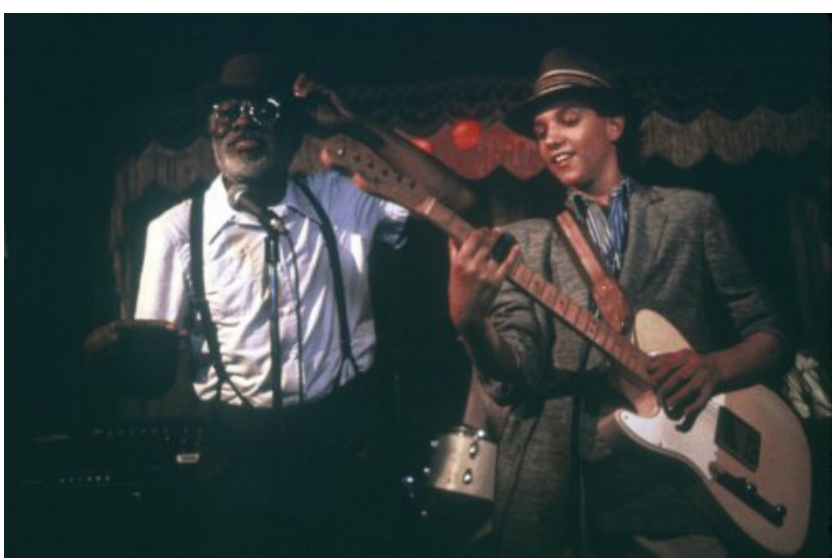

Su contrincante improvisa complicadas melodías con mucha destreza técnica a las que Eugene, responde con suma seguridad. En su turno de improvisar y desafiar a su contrincante ocurre algo inesperado. Reinventa aquella obra de Mozart en la que anticipó, todavía sin saberlo, la puesta en marcha de su periplo. Ya no ante ese maestro "atontador" (Ranciere), infalible, sino frente a esa soledad sin referencias. 
$\mathrm{Y}$ es con este concierto reinventado y ejecutado con maestría con el que derrota al diablo. La técnica es virtuosa en ambos, pero Eugene, aún con su alma intacta, logra, como bien describe Schoenberg, tomar ese impulso que subyace en la obra de los grandes. Ha logrado bucear en lo profundo de su ser y expresarse a sí mismo (Schoenberg, 1979: xviii).

En la escena final, Eugene y Willie caminan juntos por una carretera desolada. Y mantienen el siguiente diálogo, que cierra el film y abre una brecha en el alma del espectador:

-Willie: Después que te muestre Chicago tu seguirás sin mí.

-Eugene: ¿Por qué no puedo ir contigo?, después podemos ir a Los Angeles...

- Willie: Así no son las canciones. Toma la música de alguna parte. Tómala más allá de donde la encuentres. Porque eso fue lo que hicimos. ¿Tenemos un trato?

\section{Referencias}

Daneri, C. (2000). Prohibición de EticArte. En Michel Fariña, J. y Gutiérrez, C.: Etica y cine. Eudeba: Buenos Aires.

Ariel, A (1994). El Estilo y el Acto. Manantial: Buenos Aires.

Schoenberg, A. (1979): Tratado de Armonía. Editorial Real Musical. 